\title{
CRITERIA FOR THE DIVERGENCE OF PAIRS OF TEICHMÜLLER GEODESICS
}

\author{
ANNA LENZHEN AND HOWARD MASUR
}

\begin{abstract}
We study the asymptotic geometry of Teichmüller geodesic rays. We show that, when the transverse measures to the vertical foliations of the quadratic differentials determining two different rays are topologically equivalent, but are not absolutely continuous with respect to each other, the rays diverge in Teichmüller space.
\end{abstract}

\section{INTRODUCTION}

Let $S$ be an oriented surface of genus $g$ with $n$ punctures. We assume $3 g-3+n \geq 1$. Let $\mathcal{T}(S)$ denote the Teichmüller space of $S$ with the Teichmüller metric $d(\cdot, \cdot)$. A basic question in geometry is to study the long term behavor of geodesics. In this paper we study the question of when a pair of geodesic rays $X_{1}(t), X_{2}(t)$, with possibly distinct basepoints, stay bounded distance apart, and when they diverge in the sense that $d\left(X_{1}(t), X_{2}(t)\right) \rightarrow \infty$ as $t \rightarrow \infty$.

Teichmüller's theorem implies that a Teichmüller geodesic ray is determined by a quadratic differential $q$ at the base point and that there are quadratic differentials $q(t)$ on $X(t)$ along the ray found by stretching along the horizontal trajectories of $q$ and contracting along the vertical trajectories.

Many cases of the question of divergence of rays are already known. It is a general principle that the asymptotic behavior of the ray is determined by the properties of the vertical foliation of $q$. The first instance is if the quadratic differentials $q_{1}, q_{2}$ defining the geodesic rays $X_{1}(t), X_{2}(t)$ are Strebel differentials. This means that their vertical trajectories are closed and decompose the surface into cylinders. In [7] it was shown that if the homotopy classes of the cylinders for $q_{1}$ coincide with those of $q_{2}$, then $X_{1}(t), X_{2}(t)$ stay bounded distance apart. In particular this showed that the Teichmüller metric was not negatively curved in the sense of Busemann. A second known case is if the vertical foliations of $q_{1}, q_{2}$ are the same uniquely ergodic foliation. In that case the rays also stay bounded distance apart ([8]).

The next possibility is the vertical foliations of $q_{1}, q_{2}$ are topologically equivalent, have a minimal component and yet are not uniquely ergodic. (It is well-known that for any quadratic differential, the vertical trajectories decompose the surface into cylinders and subsurfaces in which every trajectory is dense). In that case in each minimal component there exist a finite number of mutually singular ergodic measures, and any transverse

Date: March 17, 2009.

The second author is supported in part by the NSF. 
measure is a convex combination of the ergodic measures. Ivanov ([5]) showed that if the transverse measures of $q_{1}, q_{2}$ in these minimal components are absolutely continuous with respect to each other, then the rays stay bounded distance apart. In this paper we prove the converse.

Let $q_{1}, q_{2}$ quadratic differentials on $X_{1}$ and $X_{2}$ with vertical foliations $\left[F_{q_{1}}^{v},\left|d x_{1}\right|\right]$ and $\left[F_{q_{2}}^{v},\left|d x_{2}\right|\right]$ and determining rays $X_{1}(t), X_{2}(t)$. Our main result is then

Theorem A. Suppose $F_{q_{1}}^{v}$ and $F_{q_{2}}^{v}$ are topologically equivalent. Suppose there is a minimal component $\Omega$ of the foliations $F_{q_{i}}^{v}$ with ergodic measures $\nu_{1}, \ldots, \nu_{p}$ and so that restricted to $\Omega,\left|d x_{1}\right|=\sum_{i=1}^{p} a_{i} \nu_{i},\left|d x_{2}\right|=\sum_{i=1}^{p} b_{i} \nu_{i}$ and there $i s$ some index $i$ so that either $a_{i}=0$ and $b_{i}>0$, or $a_{i}>0$ and $b_{i}=0$. Then the rays $X_{1}(t)$ and $X_{2}(t)$ diverge.

In particular, this holds when the transverse measures are distinct ergodic measures.

The last possibility is that the vertical foliations of $q_{1}, q_{2}$ are not topologically equivalent. If the geometric intersection of the vertical foliations is nonzero, then the rays diverge $([5])$. We prove

Theorem B. Suppose $q_{1}, q_{2}$ are quadratic differentials such that the vertical foliations $\left[F_{q_{1}}^{v},\left|d x_{1}\right|\right]$ and $\left[F_{q_{2}}^{v},\left|d x_{2}\right|\right]$ are not topologically equivalent, but $i\left(\left[F_{q_{1}}^{v},\left|d x_{1}\right|\right],\left[F_{q_{2}}^{v},\left|d x_{2}\right|\right]\right)=0$. Then the rays $\left.X_{1}(t)\right)$ and $X_{2}(t)$ diverge.

These theorems together with the previously known results completely answer the question of divergence of rays.

The outline of the proof of Theorem $\mathrm{A}$ is as follows. In Proposition 1, we will show that, for the flat metrics defined by the quadratic differentials $q_{1}(t)$ and $q_{2}(t)$, for any sufficiently large time $t$, there is a subsurface $Y(t) \subset \Omega$ with its area small in one metric and bounded away from zero in the other, while its boundary is short in both metrics. This is where we use the assumption that the measures are not absolutely continuous with respect to each other. We will then apply Lemma 6 to find a bounded length curve $\gamma(t) \subset Y(t)$ which is "mostly vertical" with respect to the metric of $q_{1}(t)$. It has comparable length in the metric of $q_{2}(t)$. Using the fact that the quadratic differentials give comparable length to $\gamma(t)$ while giving very different areas to $Y(t)$, Lemma 7 will allow us to show that the ratio of the extremal length of $\gamma(t)$ along one ray to the extremal length on the other is large. We then apply Kerckhoff's formula to conclude that the surfaces are far apart in Teichmuller space.

We will also prove

Theorem C. Let $\nu_{1}, \ldots, \nu_{p}$ be maximal collection of ergodic measures for a minimal foliation $[F, \mu]$. Then there is a sequence of multicurves $\gamma_{n}=\left\{\gamma_{n}^{1}, \ldots, \gamma_{n}^{k}\right\}$ such that $\gamma_{n}^{j} \rightarrow\left[F, \nu_{j}\right]$ in $\mathcal{P} \mathcal{M F}$.

In other words, any two topologically equivalent measured foliations can be approximated by a sequence of multicurves, with possibly different weights. This result settles a question asked by Moon Duchin.

Acknowledgements. We would like to thank Moon Duchin, Ursula Hamenstadt, and Kasra Rafi for useful discussions and their interest in this project. We would also 
like to thank the referee for numerous helpful suggestions. We are also grateful to the Mathematical Sciences Research Institute for the support and hospitality during the time this research was conducted.

\section{BACKGROUND}

2.1. Measured foliations. Recall a measured foliation on a surface $S$ consists of a finite set $\Sigma$ of singular points and a covering of $S \backslash \Sigma$ by open sets $\left\{U_{\alpha}\right\}$ with charts $\phi_{\alpha}: U_{\alpha} \rightarrow \mathbb{R}^{2}$ such that the overlap maps are of the form

$$
(x, y) \rightarrow( \pm x+c, f(x, y)) .
$$

The leaves of the foliation are the lines $x=$ constant. The points $\Sigma$ are $p$-pronged singularities for $p \geq 3$. One allows single pronged singularities at the punctures. A measured foliation comes equipped with a transverse invariant measure which in the above coordinates is given by $\mu=|d x|$. Henceforth we will denote measured foliations by $[F, \mu]$. We will write $F$ to denote a (topological) foliation when we are ignoring the measure.

For the rest of the paper, a curve will always mean a simple closed curve. For any homotopy class of simple closed curves $\beta$, let

$$
i([F, \mu], \beta)=\inf _{\beta^{\prime} \sim \beta} \int_{\beta^{\prime}} d \mu .
$$

The intersection with simple closed curves extends to an intersection function

$$
i\left(\left[F_{1}, \mu_{1}\right],\left[F_{2}, \mu_{2}\right]\right)
$$

on pairs of measured foliations. Thurston's space of measured foliations is denoted $\mathcal{M} F$ and the projective space of measured foliations by $\mathcal{P} M F$.

Let $\Gamma_{F}$ denote the compact leaves of $F$ joining singularities. It is well-known that each component $\Omega$ of $S \backslash \Gamma_{F}$ is either an annulus swept out by closed leaves or a minimal domain in which every leaf is dense.

Definition 1. We say that two foliations $F_{1}, F_{2}$ on $S$ are topologically equivalent, and we write $F_{1} \sim F_{2}$, if there is a homeomorphism of $S \backslash \Gamma_{F_{1}} \rightarrow S \backslash \Gamma_{F_{2}}$ isotopic to the identity which takes the leaves of $F_{1}$ to the leaves of $F_{2}$.

Note that this definition does not refer to the measures.

Definition 2. A foliation $[F, \mu]$ in a minimal domain $\Omega$ is said to be uniquely ergodic if the transverse measure $\mu$ restricted to $\Omega$ is the unique transverse measure of the foliation $F$ up to scalar multiplication.

More generally, suppose $\Omega$ is a minimal component of $[F, \mu]$. There exist invariant transverse measures $\nu_{1}=\nu_{1}(\Omega), \ldots, \nu_{p}=\nu_{p}(\Omega)$ such that

- $p$ is bounded in terms of the topology of $\Omega$.

- $\nu_{i}$ is ergodic for each $i$.

- any transverse invariant measure $\nu$ on $\Omega$ can be written as $\nu=\sum_{i=1}^{p} a_{i} \nu_{i}$ for $a_{i} \geq 0$. 
Thus the transverse measures are parametrized by a simplex in $\mathbb{R}^{p}$. Two foliations, $\left[F, \mu_{1}\right]$ and $\left[F, \mu_{2}\right]$, are absolutely continuous with respect to each other if, when the measures are expressed as a convex combination as above, the indices with positive coefficients are identical. Equivalently, they are absolutely continuous with respect to each other if they lie in the same open face of the simplex.

2.2. Quadratic differentials and Teichmüller rays. A meromorphic quadratic differential $q$ on a closed Riemann surface $X$ with a finite number of punctures removed is a tensor of the form $q(z) d z^{2}$ where $q$ is a holomorphic function and $q(z) d z^{2}$ is invariant under change of coordinates. We allow $q$ to have at most simple poles at the punctures.

As such there is a metric defined by $|q(z)|^{1 / 2}|d z|$. The length of an arc $\beta$ with respect to the metric will be denoted by $|\beta|_{q}$. There is an area element defined by $|q(z)||d z|^{2}$. We will denote by Area $_{q} \Omega$ the area of a subsurface $\Omega \subseteq X$.

Away from the zeroes and poles of $q$ there are natural holomorphic coordinates $z=x+i y$ such that in these coordinates $q=d z^{2}$. The lines $x=$ constant with transverse measure $|d x|$ define the vertical foliation $\left[F_{q}^{v},|d x|\right]$. The lines $y=$ constant with transverse measure $|d y|$ define the horizontal measured foliation $\left[F_{q}^{h},|d y|\right]$. The transverse measure of an arc $\beta$ with respect to $|d y|$ will be denoted by $v_{q}(\beta)$ and called the vertical length of $\beta$. Similarly, we have the horizontal length denoted by $h_{q}(\beta)$. The area element in the natural coordinates is given by $d x d y$.

We denote by $\Gamma_{q}=\Gamma_{F_{q}^{v}}$ the vertical critical graph of $q$. This is the union of the vertical leaves joining the zeroes of $q$.

The Teichmüller space of $S$ denoted by $\mathcal{T}(S)$ is the set of equivalence classes of homeomorphisms $f: S \rightarrow X$ where $f_{i}: S \rightarrow X_{i}, i=1,2$ are equivalent if there is a conformal map $h: X_{1} \rightarrow X_{2}$ with $f_{2}$ homotopic to $h \circ f_{1}$. The Teichmüller metric on $\mathcal{T}(S)$ is the metric defined by

$$
d\left(\left(X_{1}, g_{1}\right),\left(X_{2}, g_{2}\right)\right):=\frac{1}{2} \inf \left\{\log K(f): f: X_{1} \rightarrow X_{2} \text { is homotopic to } g_{2} \circ g_{1}^{-1}\right\}
$$

where $f$ is quasiconformal and

$$
K(f):=\left\|K_{x}(f)\right\|_{\infty} \geq 1
$$

is the quasiconformal dilatation of $f$, where

$$
K_{x}(f):=\frac{\left|f_{z}(x)\right|+\left|f_{\bar{z}}(x)\right|}{\left|f_{z}(x)\right|-\left|f_{\bar{z}}(x)\right|}
$$

is the pointwise quasiconformal dilatation at $x$.

Teichmüller's Theorem states that, given any $X_{1}, X_{2} \in \mathcal{T}(S)$, there exists a unique (up to translation in the case when $S$ is a torus) quasiconformal map $f$, called the Teichmüller map, realizing $d\left(X_{1}, X_{2}\right)$. The Beltrami coefficient $\mu_{f}:=\frac{\bar{\partial} f}{\partial f}$ is of the form $\mu_{f}=k \frac{\bar{q}}{|q|}$ for a unique unit area quadratic differential $q$ on $X_{1}$ and some $k$ with $0 \leq k<1$. Define $t$ by

$$
e^{2 t}=\frac{1+k}{1-k}
$$


There is a quadratic differential $q(t)$ on $X_{2}$ such that in the natural local coordinates $w=u+i v$ of $q(t)$ and $z=x+i y$ of $q$ the map $f$ is given by

$$
u=e^{t} x \quad v=e^{-t} y \text {. }
$$

Thus $f$ expands along the horizontal leaves of $q$ by $e^{t}$, and contracts along the vertical leaves by $e^{-t}$.

Conversely, any unit area $q$ on $X$ determines a 1-parameter family of Teichm̈uller maps $f_{t}$ defined on $X$. Namely $f_{t}$ has Beltrami differential $\mu=k \frac{\bar{q}}{|q|}$ where $e^{2 t}=\frac{1+k}{1-k}$. The image surface is denoted by $X(t)$ and $X(t) ; t \geq 0$ is the Teichmüller ray based at $X$ in the direction of $q$. On each $X(t)$ we have the quadratic differential $q(t)$.

2.3. Extremal length and Annuli. We recall the notion of extremal length. Suppose $X$ is a Riemann surface and $\Gamma$ is a family of $\operatorname{arcs}$ on $X$. Suppose $\rho$ is a conformal metric on $X$. For an $\operatorname{arc} \gamma$, denote by $\rho(\gamma)$ its length and by $A(\rho)$ the area of $\rho$.

\section{Definition 3.}

$$
\operatorname{Ext}_{X}(\Gamma)=\sup _{\rho} \frac{\inf _{\gamma \in \Gamma} \rho^{2}(\gamma)}{A(\rho)}
$$

where the sup is over all conformal metrics $\rho$.

We will apply this definition when $\Gamma$ consists of all simple closed curves in a free homotopy class of some $\alpha$. In that case we will write $\operatorname{Ext}_{X}(\alpha)$. It is also worth noting that if $q$ is a unit area quadratic differential then $\operatorname{Ext}_{X}(\alpha) \geq|\alpha|_{q}^{2}$ since $q$ gives a competing metric. (Here again $|\alpha|_{q}$ denoted the length of the geodesic in the homotopy class of $\alpha$.)

The following formula due to Kerckhoff ([6]) is extremely useful in estimating Teichmüller distances. For $X_{1}, X_{2} \in \mathcal{T}(S)$

$$
d\left(X_{1}, X_{2}\right)=\frac{1}{2} \log \sup _{\alpha} \frac{\operatorname{Ext}_{X_{2}}(\alpha)}{\operatorname{Ext}_{X_{1}}(\alpha)}
$$

We will also need the following inequalities, comparing hyperbolic and extremal lengths. They are given by Corollary 3 in Maskit[12]. The first says that

$$
\operatorname{Ext}_{X}(\alpha) \leq \frac{1}{2} l_{\sigma}(\alpha) e^{\frac{1}{2} l_{\sigma}(\alpha)}
$$

where $l_{\sigma}(\alpha)$ repsesents the length of the geodesic, in the homotopy class of $\alpha$, with respect to the hyperbolic metric of $X$. The second says that as $l_{\sigma}(\alpha) \rightarrow 0$ we have

$$
\frac{\operatorname{Ext}_{X}(\alpha)}{l_{\sigma}(\alpha)} \rightarrow 1
$$

Definition 4. Suppose there is an embedding of a Euclidean cylinder in $\mathbb{R}^{3}$ into $X$ which is an isometry with respect to the metric of $q$. The image is called a flat cylinder. The cylinder is maximal if it cannot be enlarged. In that case there are singularities on each boundary component of the cylinder. 
We need a definition and estimates found in [3] and [14]. We first adopt the following notation. If two quantites $a$ and $b$ differ by multiplicative and additive constants that depend only on the topology, then we will often write

$$
a \asymp b \text {. }
$$

Definition 5. Given a quadratic differential with its metric $q$, an expanding annulus $A$ is an annulus where the curvature of each boundary component has constant sign, either positive or negative at each point, the boundary curves are equidistant and there are no zeroes inside $A$.

Let $d(A)$ be the distance between the boundary components of an expanding annulus. It is universally bounded. The following statement can be found as Corollary 5.4 of [3].

Lemma 1. Suppose $q$ is a quadratic differential of area 1 on $X$ with its hyperbolic metric $\sigma$, and $\beta$ is a sufficiently short curve.

$$
\frac{1}{l_{\sigma}(\beta)} \asymp \max (\operatorname{Mod}(F(\beta), \operatorname{Mod}(A(\beta)),
$$

where $F(\beta)$ is the maximal flat cylinder, $A(\beta)$ is the maximal expanding annulus with one boundary component the $q$-geodesic in the class of $\beta$ and

$$
\operatorname{Mod}(A(\beta)) \asymp \log \frac{d(A(\beta))}{|\beta|_{q}} .
$$

(3) the other boundary component of $A(\beta)$ contains a zero of $q$.

We will also need the following result from [13]. Minsky gives a useful estimate of the extremal length of a curve, which uses collar decomposition. For $0<\epsilon_{1}<\epsilon_{0}$ less than the Margulis constant, let $\mathcal{A}$ be the collection of pairwise disjoint annular neighborhoods of the geodesics of hyperbolic length at most $\epsilon_{1}$, whose internal boundary components have hyperbolic length $\epsilon_{0}$. Then the union of $\mathcal{A}$ with the collection of components of $X-\mathcal{A}$ is the $\left(\epsilon_{0}, \epsilon_{1}\right)$ collar decomposition of $X$. For a subsurface $Q \subset X$, and $\alpha$ a homotopy class of curves, we denote by $\operatorname{Ext}_{Q}(\alpha)$ the extremal length of the restriction of the curves in $\alpha$ to $Q$.

Theorem 1. [Theorem 5.1 in [13]] Let $X$ be a Riemann surface of finite type with boundary lengths in the hyperbolic metric at most $\ell_{0}$, and let $\mathcal{Q}$ be the set of components of the $\left(\epsilon_{0}, \epsilon_{1}\right)$ collar decomposition of $X$. Then, for any curve $\alpha$ in $X$, then

$$
\operatorname{Ext}_{X}(\alpha) \asymp \max _{Q \in \mathcal{Q}} \operatorname{Ext}_{Q}(\alpha)
$$

where the multiplicative factors depends only on $\epsilon_{0}, \epsilon_{1}, \ell_{0}$ and the topological type of $X$. 
2.4. Limits of quadratic differentials. We need the following convergence result for quadratic differentials where one or more curves have extremal length approaching 0 . The proof follows more or less immediately from results in [11].

Theorem 2. Suppose $X_{n}$ is a sequence of Riemann surfaces, $q_{n}$ is a sequence of unit area quadratic differentials on $X_{n}$, and $\gamma_{1}, \ldots, \gamma_{j}$ is a collection of disjoint simple closed curves such that

- the extremal length of each $\gamma_{i}$ goes to 0 along $X_{n}$

- the extremal length of every other closed curve is bounded below away from 0 along the sequence.

- there is no flat cylinder in the homotopy class of $\gamma_{i}$

Then by passing to a subsequence, for any subsurface $\Omega_{n} \subset X_{n}$ bounded by the geodesic representatives of the $\gamma_{i}$, whose $q_{n}$-area is bounded away from 0 , there is a surface $\Omega_{\infty}$ with punctures and a nonzero finite area quadratic differential $q_{\infty}$ on $\Omega_{\infty}$ such that $q_{n}$ restricted to $\Omega_{n}$ converges uniformly on compact sets to $q_{\infty}$.

The convergence means that for any neighborhood $U$ of the punctures on $\Omega_{\infty}$

(1) for large enough $n$ there is a conformal map $F_{n}: \Omega_{\infty} \backslash U \rightarrow X_{n}$

(2) $F_{n}^{*} q_{n} \rightarrow q_{\infty}$ as $n \rightarrow \infty$ uniformly on $\Omega_{\infty} \backslash U$.

Proof. Using the compactification of the moduli space of Riemann surfaces (see [2]), by passing to a subsequence we can assume $X_{n}$ converges to a limiting Riemann surface $X_{\infty}$ with paired punctures corresponding to each $\gamma_{i}$ so that (1) holds above. Then again passing to a subsequence we can assume $q_{n}$ converges to some finite area quadratic differential $q_{\infty}$ on each component $\Omega_{\infty}$ of $X_{\infty}$; the convergence as in (2). We need to show that that if $\Omega_{n}$ has $q_{n}$-area bounded below then $q_{\infty}$ is not identically 0 on the corresponding $\Omega_{\infty}$. For each paired punctures on $X_{\infty}$ pick holomorphic coordinates $0<\left|z_{i}\right|<1$ and $0<\left|w_{i}\right|<1$ on the corresponding punctured discs. For $n$ sufficiently large, for each $i$ there is a $t_{i}=t_{i}(n)$ which goes to 0 as $n \rightarrow \infty$ such that $X_{n}$ can be recovered from $X_{\infty}$ by removing the discs $0<\left|z_{i}\right|<\left|t_{i}\right|$ and $0<\left|w_{i}\right|<\left|t_{i}\right|$ and then gluing the disc $\left|t_{i}\right| \leq\left|z_{i}\right| \leq 1$ to the disc $\left|t_{i}\right| \leq\left|w_{i}\right| \leq 1$ by the formula

$$
z_{i} w_{i}=t_{i} .
$$

This produces an annulus in the class of $\gamma_{i}$. In forming $X_{n}$ we also allow a small deformation of the complex structure of $X_{\infty}$ in the complement of the union of the discs. We need to consider the punctured discs $0<\left|z_{i}\right|<1$ contained in $\Omega_{\infty}$ and the corresponding annulus

$$
A_{i}=\left\{z_{i}:\left|t_{i}\right|^{1 / 2}<\left|z_{i}\right|<1\right\} \subset X_{n} .
$$

In the coordinates of $A_{i}$ the $q_{n}$-geodesic in the class of $\gamma_{i}$ lies outside any fixed compact set $K$ for $n$ large enough. Fix now the index $i$ and suppress that subscript. By the Corollary following Lemma 5.1 in [11], we can express $q_{n}$ in $A=A_{i}$ as

$$
q_{n}=a_{n} / z^{2}+f_{n} / z+t g_{n} / z^{3}
$$

where $a_{n}, f_{n}, g_{n}$ are uniformly bounded family of holomorphic functions of $z$. It is easy to see that the last term integrated over $A$ goes to 0 as $t=t_{i}$ goes to 0 . Since there is no flat 
annulus in the class of $\gamma_{i}$, by Lemma 5.3 of [11], we have

$$
-\left|a_{n}\right|^{1 / 2} \log |t| \leq 1
$$

This implies that the first term of the expansion of $q_{n}$ also has small integral over $A$. Since we are assuming that the integral of $\left|q_{n}\right|$ is bounded away from 0 on $\Omega_{n}$ we must have that $f_{n}$ converges to a nonzero function on the disc $0<|z|<1$, and so $q_{\infty}$ is not identically 0 .

We have the following Corollary.

Corollary 1. With same assumption as in Theorem 2 suppose in addition that $Y_{n}$ is a subsurface of a complimentary component $\Omega_{n}$ of the $\gamma_{i}$ with geodesic boundary. Suppose the $q_{n}$ area of $Y_{n}$ is bounded below away from 0 and the $q_{n}$ - length of the boundary of $Y_{n}$ is bounded above. Then by passing to a subsequence there is surface $Y_{\infty}$ and a nonzero quadratic differential $q_{\infty}$ on $Y_{\infty}$ of finite area such that $q_{n}$ restricted to $Y_{n}$ converges to $q_{\infty}$ on $Y_{\infty}$, uniformly on compact sets.

Proof. We apply Theorem 2 to find $q_{\infty}$ on $\Omega_{\infty}$ such that $q_{n}$ restricted to $\Omega_{n}$ converges uniformly on compact sets to $q_{\infty}$. Since the boundary of $Y_{n} \subset \Omega_{n}$ has bounded length, on any boundary component of $Y_{n}$ not among the $\gamma_{i}, q_{n}$ converges to $q_{\infty}$. This gives the Corollary.

\section{Lemmas RELATing LENGTH, SLOPE AND AREA}

We need to recognize instances when the area of a subsurface is small. In the first Lemma we show that if all bounded length curves have small horizontal length, then the area is small.

Lemma 2. For all $\epsilon>0, D>0$, there exists $\delta>0, C>0$ such that for all $C_{1}>C$, if $q$ is a quadratic differential on a subsurface $\Omega$ which does not contain a flat cylinder parallel to a boundary component and which has the properties that

- $|\partial \Omega|_{q} \leq D$

- every closed geodesic $\alpha \subset \Omega$ satisfying $|\alpha|_{q} \leq C_{1}$ also satisfies $h_{q}(\alpha)<\delta$.

Then $\operatorname{Area}_{q}(\Omega) \leq \epsilon$.

Proof. We argue by contradiction. If the Lemma is not true, there exists $\epsilon, D$ and a sequence of quadratic differentials $q_{n}$ on surfaces $\Omega_{n}$ such that $\left|\partial \Omega_{n}\right|_{q_{n}} \leq D$ and such that all curves $\alpha \subset \Omega_{n}$ that satisfy $|\alpha|_{q_{n}} \leq n$ also satisfy $h_{q_{n}}(\alpha)<\frac{1}{n}$ and yet $\operatorname{Area}_{q_{n}}\left(\Omega_{n}\right) \geq \epsilon$. Applying Corollary 1 , by passing to a subsequence we can assume $\Omega_{n} \rightarrow \Omega_{\infty}$ and $q_{n} \rightarrow q_{\infty}$. Now each geodesic $\alpha$ of $q_{\infty}$ has horizontal length equal to 0 which is impossible, since $\Omega_{\infty}$ is not a flat cylinder.

The next lemma compares areas of flat cylinders with respect to different flat metrics.

Lemma 3. Suppose $q_{1}, q_{2}$ are quadratic differentials with the same horizontal foliation $|d y|$ and whose vertical foliations are topologically equivalent with transverse measures $\nu_{1}, \nu_{2}$. For any $B$, there exists $\epsilon_{0}$ such that for all $\epsilon<\epsilon_{0}$, if $C_{1}=C_{1}(\beta)$ is a maximal flat cylinder for $q_{1}$ with core curve $\beta$ such that 
- The absolute value of the slope of $\beta$ in $C_{1}$ is at least 1.

- $|\beta|_{q_{1}} \leq \epsilon_{0}$.

- $\operatorname{Area}_{q_{1}}\left(C_{1}\right) \geq B$.

- Any horizontal segment $I$ crossing $C_{1}$ satisfies $\nu_{2}(I) \leq \epsilon$.

Then if $\mathrm{C}_{2}$ is the maximal flat cylinder defined by $q_{2}$ in the class of $\beta$ we have Area $q_{q_{2}}\left(C_{2}\right) \leq$ $2 \epsilon \epsilon_{0}$.

Proof. We may represent $C_{1}$ as a parallelogram with a pair of horizontal sides that are glued to each other by a translation. Let $I$ be an oriented horizontal segment crossing $C_{1}$ starting at a singularity $P_{0}$ on one boundary component. Let $Q_{0}$ be the endpoint of $I$ on the other boundary component. Assume without loss of generality that the slope of $\beta$ in $C_{1}$ is negative. This means that there is a vertical leaf through $P_{0}$ that enters $C_{1}$ in the positive direction and returns to $I$ without leaving $C_{1}$ and translated by $h_{1}:=h_{q_{1}}(\beta) \leq \epsilon_{0}$. Starting at $P_{0}$, for $\epsilon_{0}$ sufficiently small compared to $B$, there will be at least two additional returns for the vertical leaf through $P_{0}$ before the leaf leaves the cylinder. Since the vertical foliations of $q_{1}$ and $q_{2}$ coincide, the same is true for the vertical leaf of $q_{2}$ leaving $P_{0}$ although now the translation amount, denoted $h_{2}$, is different.

Given any three consecutive intersections with $I$ of the leaf starting at $P_{0}$, there is a closed geodesic with respect to $q_{2}$ homotopic to $\beta$ through the middle point on $I$. Thus $C_{1}$ contains closed geodesics, with respect to the flat structure of $q_{2}$, homotopic to $\beta$. That is, there is a maximal flat cylinder $C_{2}$ some of whose core curves are contained in $C_{1}$. Since maximal cylinders have singularities on their boundaries, either $P_{0}$ is on the boundary of $C_{2}$ or possibly outside it. It is possible that $Q_{0}$ is in the interior of $C_{2}$, so if we take the closed geodesic $\beta$ of $q_{2}$ through $Q_{0}$ it does not pass through a singularity. However in that case, if we similarly take a horizontal segment $I^{\prime}$ crossing $C_{1}$ starting at a singularity $P_{1}$ on the same boundary component of $C_{1}$ as $Q_{0}$, then $\beta$ cuts $I^{\prime}$ in its interior. This implies that the horizontal distance across $C_{2}$ is at most $\nu_{2}(I)+\nu_{2}\left(I^{\prime}\right) \leq 2 \epsilon$. Since the height of $\beta$ is at most $\epsilon_{0}$, we get the desired area bound for $C_{2}$.

The next Lemma gives a lower bound of extremal length of a curve family in terms of the area of the surface it is contained in and the length of the boundary.

Lemma 4. Let $X$ be a Riemann surface. Let $q$ be a unit area quadratic differential on $X$. Let $\Omega$ be a subsurface with geodesic boundary and which does not contain a flat cylinder parallel to a boundary component. If the length $|\partial \Omega|_{q}$ is small enough, then for any homotopy class of curves $\alpha \subset \Omega$ with geodesic representative $\alpha$,

$$
\operatorname{Ext}_{X}(\alpha) \geq \frac{|\alpha|_{q}^{2}}{\operatorname{Area}_{q}(\Omega)+O\left(|\partial \Omega|_{q}^{2}\right)} .
$$

Proof. Note that this lemma does not simply follow from the definition of extremal length since the area of $\Omega$ may be smaller than 1. Let $\epsilon=|\partial \Omega|_{q}$. Define a metric $\rho$ on $X$ as folows. Let $\rho$ coincide with the $q$-metric on $\mathcal{N}_{\epsilon}(\Omega)$, the $\epsilon$-neighborhhood of $\Omega$ and the $q$ metric multiplied by a small $\delta$ on $\Omega^{\prime}=X \backslash \mathcal{N}_{\epsilon}(\Omega)$. Let $\alpha^{\prime \prime}$ be any curve in the homotopy 
class of $\alpha$. If $\alpha^{\prime \prime}$ is not contained in $\Omega$ then $\alpha^{\prime \prime}$ and a segment of $\partial \Omega$ bound a disk. The fact that $d_{\rho}\left(\Omega^{\prime}, \Omega\right)=\epsilon$ and $\partial \Omega$ is a geodesic implies that we can replace an arc of $\alpha^{\prime \prime}$ with an arc of $\partial \Omega$ to produce $\alpha^{\prime \prime \prime} \subset \bar{\Omega}$ with smaller length. We conclude that the infimum of the length in the metric $\rho$ is realized by the geodesic $\alpha$ in $\Omega$. By definition,

$$
\operatorname{Ext}_{X}(\alpha) \geq \frac{\inf _{\alpha^{\prime \prime} \sim \alpha} \rho\left(\alpha^{\prime \prime}\right)_{q}^{2}}{A(\rho)} \geq \frac{|\alpha|_{q}^{2}}{\operatorname{Area}_{q}(\Omega)+O\left(\epsilon^{2}\right)+\delta \operatorname{Area}_{q}\left(\Omega^{\prime}\right)} .
$$

The term $O\left(\epsilon^{2}\right)$ in the inequality above comes from $\operatorname{Area}_{q}\left(\mathcal{N}_{\epsilon}(\Omega) \backslash \Omega\right)$. Since $\delta$ is arbitrary, we have the result.

Definition 6. Given a quadratic differential $q$ and $\delta>0$, a geodesic $\gamma$ in the $q$ metric is called almost $(q, \delta)$-vertical if $v_{q}(\gamma) \geq \delta h_{q}(\gamma)$.

Note that $\delta$ may be small in the above definition.

Lemma 5. Let $q$ be a quadratic differential on $X$ a surface without boundary. For any $\delta>0$ there is a curve $\beta$ which is almost $(q, \delta)$-vertical.

Proof. If $\Gamma_{q} \neq \emptyset$ there is a vertical saddle connection which is obviously almost $(q, \delta)$ vertical. If a vertical leaf is dense in a subsurface then the boundary of the subsurface contains a vertical saddle connection. Thus we can assume that the vertical foliation is minimal. Let $A$ be the area of $q$. The first return map of the foliation to a horizontal transversal $I$ with an endpoint at a singularity defines a generalized interval exchange transformation. Choose a horizontal transversal $I$ of length $\lambda$ satisfying

$$
\lambda^{2}<\frac{A}{\delta}
$$

The transversal $I$ determines a decomposition of the surface into rectangles $\left\{R_{i}\right\}$, with heights $h_{i}$ and widths $\lambda_{i}$, whose horizontal sides are subsets of $I$. Each rectangle has two horizontal sides on $I$. Consequently, if we count each $\lambda_{i}$ twice we have

$$
\sum_{i} \lambda_{i}=2 \lambda
$$

Since we count each $\lambda_{i}$ twice we have

$$
\sum_{i} h_{i} \lambda_{i}=2 A
$$

We conclude that

$$
\max _{i} h_{i} \geq \frac{A}{\lambda}
$$

Let $h_{i}$ realize this maximum. There are two cases. The first case (see Figure 1 ) is that the horizontal sides of $R_{i}$ are on opposite sides of $I$. Fix a small neighborhood $\mathcal{N}$ of $I$. We form a simple closed curve $\beta=\beta_{1} * \beta_{2}$. Here $\beta_{1}$ is a vertical segment in $R_{i}$ whose endpoints $p$ and $q$ are on the boundary of $\mathcal{N}$, and $\beta_{2}$ is an arc transverse to the horizontal foliation in $\mathcal{N}$ joining $p$ and $q$. Then $\beta$ is also transverse to the horizontal foliation. Its geodesic 


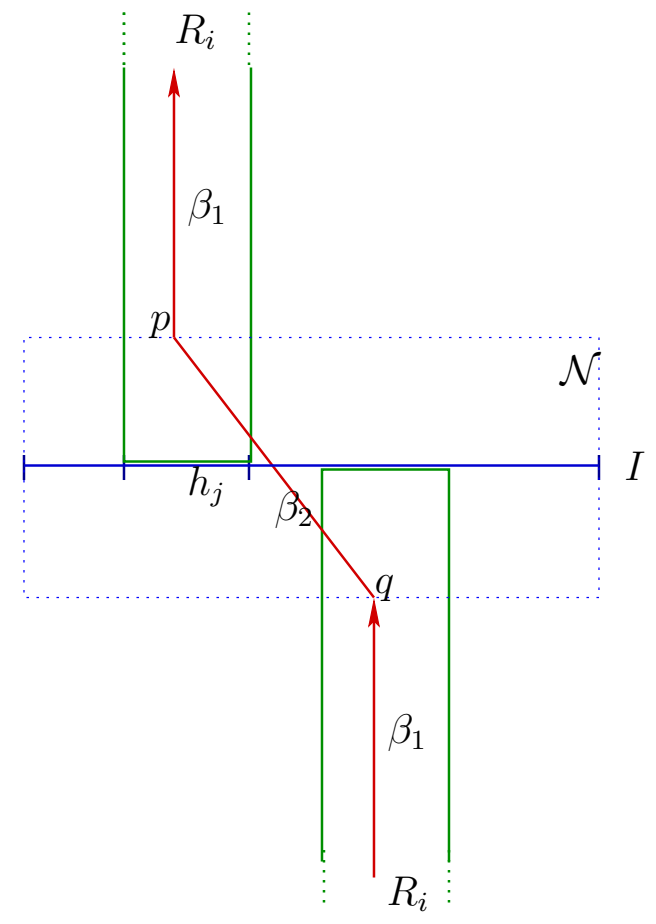

Figure $1 . \beta$ is the union of $\beta_{1}$ and $\beta_{2}$

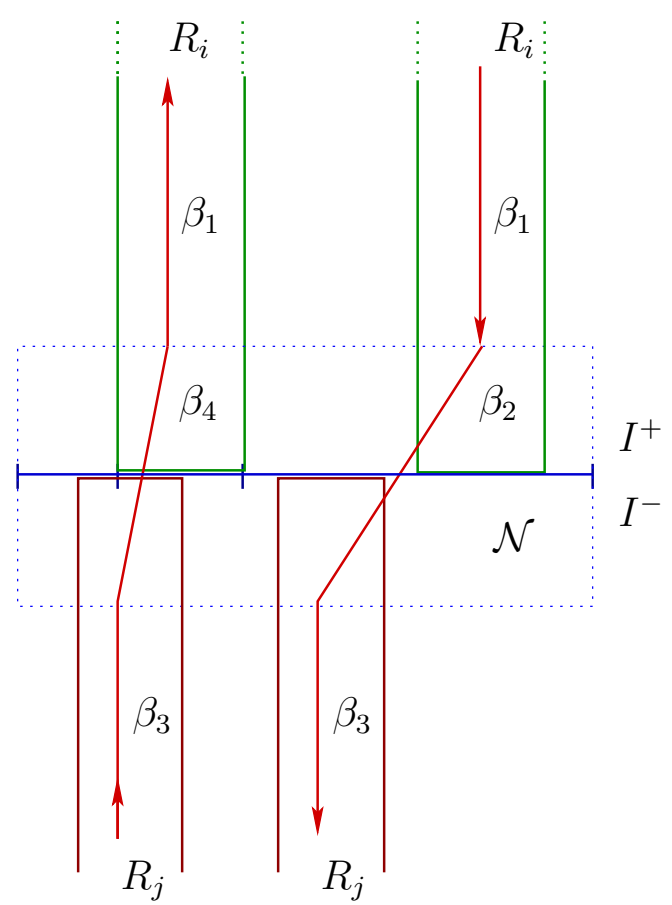

FiguRE $2 . \beta$ is the union of $\beta_{1}, \beta_{2}, \beta_{3}$ and $\beta_{4}$

representative has the same vertical length as $\beta$, namely, $h_{i}$. The horizontal length of $\beta$ is at most $\lambda$. Together with (2) and (3) we have that

$$
\frac{v_{q}(\beta)}{h_{q}(\beta)} \geq \frac{h_{i}}{\lambda} \geq \frac{A}{\lambda^{2}} \geq \delta .
$$

In the second case (Figure 2), both horizontal sides of $R_{i}$ are on the same side of $I$ (call it $I^{+}$). Then there must also be a rectangle $R_{j}$ with top and bottom on $I_{-}$. We may form a simple closed curve $\beta$ which consists of a vertical segment in $R_{i}$, a vertical segment in $R_{j}$ and a pair of $\operatorname{arcs}$ in $\mathcal{N}$ which are transverse to the horizontal foliation. Similar to the case above, the ratio of vertical and horizontal components of $\beta$ is at least $\delta$.

Definition 7. Given a unit area quadratic differential $q$ on a surface $X$ without boundary, a subsurface $\Omega \subsetneq X$ is said to be $\left(\epsilon, \epsilon_{0}\right)$-thick if the following conditions hold:

- $\partial \Omega$ is a geodesic in the metric of $q$.

- $\operatorname{Ext}_{X}(\partial \Omega) \leq \epsilon$

- the shortest non-peripheral curve in $\Omega$ has q length at least $\epsilon_{0}$.

The surface $X$ itself is $\epsilon_{0}$-thick if it satisfies the third condition above.

The following Lemma says that we can find almost $(q, \delta)$ vertical saddle connections in thick surfaces. 
Lemma 6. For any $B>0, \epsilon_{0}>0$ there exists $\epsilon>0, \delta>0, D>0$ and $m_{0}$ such that for any $\left(\epsilon, \epsilon_{0}\right)$-thick surface $\Omega \subset X$ which does not contain a flat annulus isotopic to a boundary component and such that $\operatorname{Area}_{q}(\Omega) \geq B$ the following two conditions hold.

(1) there is an almost $(q, \delta)$-vertical geodesic $\gamma$ whose interior lies in $\Omega$ and such that $|\gamma|_{q}<D$.

(2) For any saddle connection $\gamma$ which is not vertical or horizontal, there is an $m \leq m_{0}$ and a collection $\omega_{1}, \ldots, \omega_{m}$ of disjoint vertical segments so that for every horizontal leaf $H$

$$
\left|\operatorname{card}(H \cap \gamma)-\sum_{i=1}^{m} \operatorname{card}\left(H \cap \omega_{i}\right)\right| \leq 2 .
$$

Proof. For the proof of the first statement, we argue by contradiction. Suppose the statement is not true. Then there is a sequence $X_{n}$ of surfaces, a sequence of unit area quadratic differentials $q_{n}$ on $X_{n}$ and a sequence of $\left(1 / n, \epsilon_{0}\right)$-thick proper subsurfaces $\Omega_{n}$ with $q_{n}$-area at least $B$ such that the shortest almost $\left(q_{n}, 1 / n\right)$-vertical curve on $\Omega_{n}$ has length at least $n$. We now apply Corollary 1 to find a subsequence $q_{n}$ which converges uniformly on compact sets to $q_{\infty}$ on a limiting surface $\Omega_{\infty}$. The uniform convergence implies that $\Omega_{\infty}$ is $\epsilon_{0} / \sqrt{B}$-thick.

By Lemma 5 , taking $\delta=1$, there is a simple closed curve $\beta$ on $\Omega_{\infty}$ such that

$$
\frac{v_{q_{\infty}}(\beta)}{h_{q_{\infty}}(\beta)} \geq 1
$$

By uniform convergence, $v_{q_{n}}(\beta) \rightarrow v_{q_{\infty}}(\beta)$, and $h_{q_{n}}(\beta) \rightarrow h_{q_{\infty}}(\beta)$ and thus for large enough $n$,

$$
\frac{v_{q_{n}}(\beta)}{h_{q_{n}}(\beta)} \geq 1 / 2
$$

and furthermore, $|\beta|_{q_{n}} \leq|\beta|_{q_{\infty}}+1$. This is a contradiction to the assumption that the shortest $\left(q_{n}, 1 / n\right)$-vertical curve has length at least $n$, proving the first statement.

We prove the second statement. Begin at one endpont $p$ of $\gamma$ and take the vertical leaf $\ell_{1}$ leaving $p$ such that $\gamma$ lies in the $\pi / 2$ sector between $\ell_{1}$ and a horizontal leaf leaving $p$. Move along $\ell_{1}$ as far as possible to a point $x_{1}$ in such a way that the segment $\omega_{1}$ of $\ell_{1}$, from $p$ to $x_{1}$, a horizontal segment $\kappa_{1}$ from $x_{1}$ to a point $y_{1} \in \gamma$ and the segment $\gamma_{1}$ of $\gamma$ from $p$ to $y_{1}$ bounds an embedded triangle $\Delta_{1}$ with no singularity in its interior. If $\gamma_{1}=\gamma$ we are done. We take $\omega_{1}$ as the desired vertical segment. If not, then there is a singularity $p_{1}$ in the interior of $\kappa_{1}$. At $p_{1}$ one vertical leaf enters $\Delta_{1}$. Choose the vertical leaf $\ell_{2}$ at $p_{1}$ that makes an angle of $\pi$ with the vertical leaf that enters $\Delta_{1}$ and such that the horizontal leaf $\kappa_{1}$ through $p_{1}$ on the side of $\Delta_{1}$ is between them. Then horizontal leaves through points on $\ell_{2}$ near $p_{1}$ will intersect $\gamma$ before returning to $\ell_{2}$. Now repeat the procedure with $\ell_{2}$ in place of $\ell_{1}$ and find a maximal embedded quadrilateral $\Delta_{2}$ disjoint from $\Delta_{1}$ in its interior consisting of a pair of horizontal sides, a segment of $\gamma$ and a segment $\omega_{2} \subset \ell_{2}$. We repeat this procedure, if necessary with a new $\ell_{3}$ until the last segment on $\gamma$ ends at the other endpoint. There are a fixed number of singularities, hence a fixed number of horizontal 
sides leaving them and so a bounded number of such embedded quadrilaterals. Say the bound is $m_{0}$. The desired vertical segments are $\omega_{1}, \ldots, \omega_{m}$, where $m \leq m_{0}$.

For the sequel we will need the following result, due to Rafi, [15] relating hyperbolic and flat lengths of curves in a thick subsurface. The first statement is Theorem 1, the second is part of Theorem 4 in [15]

Theorem 3. For every $\left(\epsilon, \epsilon_{0}\right)$-thick subsurface $Y$ of a Riemann surface $X$ with hyperbolic metric $\sigma$ and quadratic differential $q$, there exists $\lambda=\lambda(q, Y)$ such that up to multiplicative constants depending only on topology of $X$

(1) For every non-periferal simple closed curve $\alpha$ in $Y$,

$$
|\alpha|_{q} \asymp \lambda l_{\sigma}(\alpha)
$$

the multiplicative constants depending only on the topology of $Y$.

(2) $\operatorname{Area}_{q}(Y) \leq \lambda^{2}$

We will now compare extremal lengths of curves that are contained in the "same" subsurface $\Omega$ measured with respect to the metrics defined by two different quadratic differentials $q_{1}, q_{2}$ on surfaces $X_{1}, X_{2}$. Specifically if $\Omega$ is a subsurface with geodesic boundary with respect to $q_{1}$, and $\Omega$ does not contain a flat cylinder isotopic to a boundary component, then we denote by $\Omega \subset X_{2}$ the subsurface containing the same set of simple closed curves and with geodesic boundary with respect to $q_{2}$. If $\Omega$ is a flat cylinder with respect to $q_{1}$, then we denote by $\Omega$ the maximal flat cylinder (possibly empty) with respect to $q_{2}$.

The following Lemma allows us to find curves with very different extremal length if a subsurface $\Omega$ has very different areas with respect to two quadratic differentials and one of the surfaces is thick.

Lemma 7. For any $B, M, \delta, \epsilon_{0}>0$, there exist $\epsilon, C, D>0$ so that the following holds. If $q_{1}$ and $q_{2}$ are quadratic differentials on $X_{1}, X_{2}$, and $\Omega$ is a proper subsurface with geodesic boundary with respect to each quadratic differential, which does not contain a flat cylinder with respect to $q_{1}$ parallel to a boundary component and such that $\Omega$ satisfies

(i)

$$
\operatorname{Area}_{q_{1}}(\Omega) \geq B, \operatorname{Area}_{q_{2}}(\Omega)<\epsilon
$$

(ii) for any almost $\left(q_{1}, \delta\right)$-vertical curve $\gamma \subset \Omega$ that satisfies $|\gamma|_{q_{1}} \leq D$, the vertical components satisfy

$$
\frac{1}{C} \leq \frac{v_{q_{1}}(\gamma)}{v_{q_{2}}(\gamma)} \leq C
$$

(iii) $|\partial \Omega|_{q_{2}}<\epsilon$

(iv) $\Omega$ is $\left(\epsilon, \epsilon_{0}\right)$ - thick with respect to $q_{1}$

then there exists a curve $\gamma$ in $\Omega$ so that

$$
\frac{\operatorname{Ext}_{X_{2}}(\gamma)}{\operatorname{Ext}_{X_{1}}(\gamma)} \geq M
$$


Proof. By Lemma 6, for some $\delta$ and $D$ there is an almost $\left(q_{1}, \delta\right)$-vertical curve $\gamma \subset \Omega$ such that

$$
\epsilon_{0} \leq|\gamma|_{q_{1}}<D
$$

Let $\sigma_{i}$ be the hyperbolic metric on $X_{i}$ and $l_{\sigma_{i}}(\gamma)$ denote the length of the geodesic $\gamma$ in the hyperbolic metric. Set $A=\operatorname{Area}_{q_{1}}(\Omega)$. By Theorem 3,

$$
l_{\sigma_{1}}(\gamma)<C_{1}|\gamma|_{q_{1}} / \sqrt{A} \leq C_{1} D / \sqrt{A}
$$

where the constant $C_{1}$ depends only on the topology of the surface. Also by Maskit's comparison of hyperbolic and extremal lengths,

$$
\operatorname{Ext}_{X_{1}}(\gamma) \leq \frac{1}{2} l_{\sigma_{1}}(\gamma) e^{l_{\sigma_{1}}(\gamma) / 2} \leq C_{1} D / \sqrt{A} e^{C_{1} D / 2 \sqrt{A}}
$$

Set $C_{2}=C_{1} D / \sqrt{A} e^{C_{1} D / 2 \sqrt{A}}$, so that

$$
\operatorname{Ext}_{X_{1}}(\gamma) \leq C_{2}
$$

On the other hand, by (4), assumption (ii) and the fact that $\gamma$ is almost $\left(q_{1}, \delta\right)$-vertical

$$
|\gamma|_{q_{2}} \geq v_{q_{2}}(\gamma)>\frac{1}{C} v_{q_{1}}(\gamma)>\frac{\delta}{C(1+\delta)}|\gamma|_{q_{1}} \geq \frac{\epsilon_{0} \delta}{C(1+\delta)}
$$

and by Lemma 4,

$$
\operatorname{Ext}_{X_{2}}(\gamma) \geq \frac{|\gamma|_{q_{2}}^{2}}{\operatorname{Area}_{q_{2}}(\Omega)+O\left(|\partial \Omega|_{q_{2}}^{2}\right)}
$$

Putting the inequalities (7), (8), (9) together and using assumptions (i) and (iii), we obtain

$$
\frac{\operatorname{Ext}_{X_{2}}(\gamma)}{\operatorname{Ext}_{X_{1}}(\gamma)} \geq \frac{\epsilon_{0}^{2} \delta^{2}}{C_{2} C^{2}(1+\delta)^{2}\left(\operatorname{Area}_{q_{2}}(\Omega)+O\left(|\partial \Omega|_{q_{2}}^{2}\right)\right)} \geq \frac{C_{3}}{\epsilon+O\left(\epsilon^{2}\right)}
$$

where $C_{3}=\frac{\epsilon_{0}^{2} \delta^{2}}{C_{2} C^{2}(1+\delta)^{2}}$. Now, setting $\epsilon$ sufficiently small compared to $\frac{C_{3}}{M}$ guarantees that the Lemma holds.

\section{Areas of Subsurfaces AlOng RAYS}

The proof of the main theorem is now based on the next proposition. We have the following set-up. Suppose $q_{1}, q_{2}$ are quadratic differentials on $X_{1}, X_{2}$ such that the vertical foliations $F_{q_{1}}^{v}, F_{q_{2}}^{v}$ are topologically equivalent and have a minimal non uniquely ergodic component $\Omega$. Suppose also that with respect to the invariant ergodic measures $\nu_{1}, \ldots, \nu_{p}$ on $\Omega,\left|d x_{1}\right|=\sum_{k=1}^{p} a_{k} \nu_{k}$, with $a_{1}>0$, while $\left|d x_{2}\right|=\sum_{k=1}^{p} b_{k} \nu_{k}$ with $b_{1}=0$. Suppose $F_{q_{1}}^{h}=F_{q_{2}}^{h}$. Let $|d y|$ denote the transverse measure to this common horizontal foliation. We normalize so that

$$
\int_{\Omega} a_{1} d \nu_{1}|d y|=1
$$


Proposition 1. With the above assumptions, let $X_{1}(t), X_{2}(t)$ be the corresponding rays, and let $q_{1}(t), q_{2}(t)$ be the quadratic differentials on $X_{1}(t), X_{2}(t)$ respectively. For any sequence of times $t_{n} \rightarrow \infty$, there is a subsequence, again denoted $t_{n}$, and constants $\epsilon_{0}>0, c>0$, so that for sufficiently small $\epsilon>0$, there is $t_{0}$, such that for $t_{n} \geq t_{0}$ there is a subsurface $Y_{1}\left(t_{n}\right) \subset \Omega$ satisfying

(i) $Y_{1}\left(t_{n}\right)$ is $\left(\epsilon, \epsilon_{0}\right)$ thick with respect to $q_{1}\left(t_{n}\right)$.

(ii) $\operatorname{Area}_{q_{1}\left(t_{n}\right)}\left(Y_{1}\left(t_{n}\right)\right) \geq a_{1}(1-c \epsilon)$.

(iii) $\operatorname{Area}_{q_{2}\left(t_{n}\right)}\left(Y_{1}\left(t_{n}\right)\right)<c \epsilon$.

Proof of Proposition 1. Since $F_{q_{1}}^{v}$ is minimal and not uniquely ergodic, we can apply Theorem 1.1 in [9], which says that the ray $X_{1}(t)$ eventually leaves every compact set in the moduli space as $t \rightarrow \infty$. (That theorem was stated in the case that the minimal component was the entire surface. The proof in the case of a minimal non uniquely ergodic component is identical. In fact the main idea of the proof is repeated below in a slightly different context). Passing to a subsequence we conclude that there exists $\gamma_{1}\left(t_{n}\right), \ldots, \gamma_{m}\left(t_{n}\right) \subset \Omega$ such that

$$
\operatorname{Ext}_{X_{1}\left(t_{n}\right)}\left(\gamma_{i}\left(t_{n}\right)\right) \rightarrow 0
$$

and such that the extremal lengths of all other curves are bounded away from 0 .

Again passing to a subsequence we can apply Theorem 2 to find $\epsilon_{0}>0$ such that for $n$ sufficiently large, there is a nonempty collection $\left\{Y\left(t_{n}\right)\right\}$ of disjoint of $\left(\epsilon, \epsilon_{0}\right)$ thick subsurfaces contained in $\Omega$. We can assume that each $Y\left(t_{n}\right)$ is either a flat annulus or it does not contain a flat annulus isotopic to a boundary component. There is a uniform bound $N$ for the number of these surfaces. Let $f_{t_{n}}: X_{1} \rightarrow X_{1}\left(t_{n}\right)$ denote the corresponding Teichmuller map.

Assume first that $Y\left(t_{n}\right)$ is not a flat cylinder. Then by passing to a subsequence, we can assume $Y\left(t_{n}\right)$ converges to a limiting punctured surface $Y_{\infty}$; the corresponding $q_{1}\left(t_{n}\right)$ converges to a limiting $q_{1, \infty}$ on $Y_{\infty}$. Thus for any neighborhood $U$ of the punctures on $Y_{\infty}$, letting $K:=Y_{\infty} \backslash U$,

(1) for large enough $n$. there is a conformal map $F_{n}: K \rightarrow Y\left(t_{n}\right)$

(2) $F_{n}^{*} q_{1}\left(t_{n}\right) \rightarrow q_{1, \infty}$ as $t_{n} \rightarrow \infty$, uniformly on $K$.

For each such $U$, for $n$ large enough, the curves $\gamma_{i}\left(t_{n}\right)$ whose lengths are approaching 0 satisfy

$$
\gamma_{i}\left(t_{n}\right) \cap F_{n}(K)=\emptyset .
$$

Since $Y\left(t_{n}\right)$ does not contain a flat cylinder in the homotopy class of a component of $\partial Y\left(t_{n}\right)$, we may find $U$ large enough so that

$$
\operatorname{Area}_{q_{1}\left(t_{n}\right)}\left(Y\left(t_{n}\right) \backslash F_{n}(K)\right) \leq \epsilon / 2 .
$$

Now, since $\nu_{i}, \nu_{j}$ are mutually singular measures, there exists $\delta>0$ and a finite set $\mathcal{I}$ of horizontal transversals $I$ to the vertical foliation in $\Omega$ such that for any $\nu_{i}, \nu_{j}, i \neq j$ there is a transversal $I_{i, j} \in \mathcal{I}$ such that

$$
\left|\nu_{i}\left(I_{i, j}\right)-\nu_{j}\left(I_{i, j}\right)\right|>\delta \text {. }
$$


Let $\Lambda_{i}$ be the set of generic points for $\nu_{i}$ and the transversals $\mathcal{I}$; that is, $\Lambda_{i}$ consists of the set of points $x$ such that, if $l_{T}(x)$ is the vertical leaf segment of $F_{q_{1}}^{v}$ through $x$ of length $T$, then for each $I \in \mathcal{I}$.

$$
\lim _{T \rightarrow \infty} \frac{1}{T} \operatorname{card}\left(l_{T}(x) \cap I\right)=\nu_{i}(I) .
$$

The sets $\Lambda_{i}$ are pairwise disjoint. With respect to the measure $\nu_{i}$, on every transversal almost every point belongs to $\Lambda_{i}$, and, with respect to the area element defined by $q_{1}$, almost every point in $\Omega$ belongs to $\cup_{i=1}^{p} \Lambda_{i}$. Let $\Lambda_{i}\left(t_{n}\right)=f_{t_{n}}\left(\Lambda_{i}\right)$.

We claim that for $n$ big enough the following holds. Let $R$ be a coordinate rectangle with respect to the flat structure of $q_{1, \infty}$ (i.e. sides are vertical and horizontal) that is contained in $K$. Then there does not exist a pair of indices $j \neq i$ and points $y_{n, i} \in f_{t_{n}}\left(\Lambda_{i}\right), y_{n, j} \in$ $f_{t_{n}}\left(\Lambda_{j}\right) ; i \neq j$ such that

$$
z_{n, i}:=F_{n}^{-1}\left(y_{n, i}\right) \in R, \quad z_{n, j}:=F_{n}^{-1}\left(y_{n, j}\right) \in R .
$$

For suppose there were points with this property. There is a coordinate rectangle $R^{\prime} \subset R$ whose vertical sides $L_{i}, L_{j}$ have endpoints at $z_{n, i}, z_{n, j}$. For every horizontal segment $H$ of $q_{1}(\infty)$

$$
\left|\operatorname{card}\left(H \cap L_{i}\right)-\operatorname{card}\left(H \cap L_{j}\right)\right| \leq 2 .
$$

Let $L_{i, n}, L_{j, n}$ be the vertical leaf segments of $q_{1}\left(t_{n}\right)$ through $y_{i, n}, y_{j, n}$ of the same length such that $F_{n}^{-1}\left(L_{i, n}\right)$ converges to $L_{i}$ and similarly with $L_{j, n}$. As $t_{n} \rightarrow \infty$, since the length of $I_{n}=f_{t_{n}}\left(I_{i, j}\right)$ goes to infinity, we have

$$
\left.\frac{1}{\operatorname{card}\left(L_{i, n} \cap I_{n}\right)} \mid \operatorname{card}\left(L_{i, n} \cap I_{n}\right)-\operatorname{card}\left(L_{j, n} \cap I_{n}\right)\right) \mid \rightarrow 0 .
$$

Mapping $L_{i, n}$ back to $X_{1}$ by $f_{t_{n}}^{-1}$, using the fact that

$$
\frac{\operatorname{card}\left(f_{t_{n}}^{-1}\left(L_{i, n}\right) \cap I_{i, j}\right)}{\left|f_{t_{n}}^{-1}\left(L_{i, n}\right)\right|}
$$

is bounded, we then have for all large $n$,

$$
\frac{1}{\left|f_{t_{n}}^{-1}\left(L_{i, n}\right)\right|}\left|\operatorname{card}\left(f_{t_{n}}^{-1}\left(L_{i, n}\right) \cap I_{i, j}\right)-\operatorname{card}\left(f_{t_{n}}^{-1}\left(L_{j, n}\right) \cap I_{i, j}\right)\right| \leq \delta / 2
$$

and we have a contradiction to (13) and (14). Thus for each rectangle $R$, there is some $i=i(R)$ such that for all $j \neq i$ and for all $x \in R$ we have

$$
\chi_{F_{n}(R)}\left(F_{n}(x)\right) \chi_{\Lambda_{j}\left(t_{n}\right)}\left(F_{n}(x)\right) \rightarrow 0 .
$$

Now we take a covering of $K$ by such rectangles. If any two rectangles $R, R^{\prime}$ overlap then $i(R)=i\left(R^{\prime}\right)$. It follows from the connectedness of $K$, that there is a single $i$ such that for all $R, i(R)=i$. Thus for $n$ large enough, for all $j \neq i,(15)$ holds. From this it follows that for $n$ large enough, for $j \neq i$

$$
\int_{Y\left(t_{n}\right)} d \nu_{j}|d y| \leq \epsilon
$$


We would like to prove an estimate similar to (16) in the case that $Y\left(t_{n}\right)$ is a flat cylinder. To do that we need a uniform version of generic points. The reason for that is that there is no natural limiting surface in the case of flat cylinders, and so the previous argument does not quite work. For each $T_{0}$, let $\Lambda_{i}^{T_{0}}$ consist of those $x \in \Lambda_{i}$ such that for $T \geq T_{0}$, for each $I \in \mathcal{I}$,

$$
\left|\frac{1}{T} \operatorname{card}\left(l_{T}(x) \cap I\right)-\nu_{i}(I)\right|<\delta / 2 .
$$

Choose $T_{0}$ so that with respect to the measure $d \nu_{i}$, except for a set of measure at most $\epsilon$, every point of $I$ belongs to $\Lambda_{i}^{T_{0}}$.

Now suppose $Y\left(t_{n}\right)$ is a flat cylinder with core curve $\beta_{n}$. Set $B=a_{1}(1-N \epsilon)$ and let $\epsilon_{0}<1$ be a constant such that Lemma 3 holds. Since $f_{t_{n}}$ is area preserving, without loss of generality, for $n$ large enough, we can also assume that at time $t_{n}$, the core curve $\beta_{n}$ is $\left(q_{1}\left(t_{n}\right), 2\right)$-almost vertical and has length smaller than $\epsilon_{0}$. This means that we can fit coordinate rectangles inside $Y\left(t_{n}\right)$ with vertical sides of length at least $\frac{1}{2}\left|\beta_{n}\right|_{q_{1}\left(t_{n}\right)}$. We can choose $n$ large enough so that $\frac{1}{2}\left|\beta_{n}\right|_{q_{1}\left(t_{n}\right)} e^{t_{n}} \geq T_{0}$. This means that if we pull back the vertical segment to $X_{1}$ its length is at least $T_{0}$ so that we can apply (17). Then the same argument given previously shows that for all but at most one $j$

$$
f_{t_{n}}\left(\Lambda_{j}^{T_{0}}\right) \cap Y\left(t_{n}\right)=\emptyset \text {. }
$$

Otherwise, for a pair $i \neq j$, we can again find a coordinate rectangle contained in the cylinder with one vertical side passing through a point of $f_{t_{n}}\left(\Lambda_{i}^{T_{0}}\right)$ and the other vertical side passing through a point of $f_{t_{n}}\left(\Lambda_{j}^{T_{0}}\right)$ and we find $\left|\nu_{i}(I)-\nu_{j}(I)\right|<\delta$, and again we have a contradiction. Thus we conclude that for $n$ large enough, for all but one $i$, for any horizontal segment crossing $Y_{1}\left(t_{n}\right)$ we have

$$
\nu_{j}(I)<\epsilon .
$$

Then except for all but at most one index $j,(16)$ also holds for flat cylinders.

Now let $Z_{1}\left(t_{n}\right)$ be the union of those $Y\left(t_{n}\right)$ such that $(16)$ holds for the index $j=1$. Then $Z_{1}(t) \subsetneq \Omega$ for otherwise we would have

$$
\int_{\Omega} d \nu_{1}|d y|<N \epsilon
$$

contradicting (11), for $\epsilon$ sufficiently small. Let $Y_{1}\left(t_{n}\right)=\Omega \backslash Z_{1}\left(t_{n}\right)$, and so we have

$$
\operatorname{Area}_{q_{1}\left(t_{n}\right)}\left(Y_{1}\left(t_{n}\right)\right) \geq a_{1} \int_{Y_{1}\left(t_{n}\right)} d \nu_{1}|d y| \geq a_{1}(1-N \epsilon)=B .
$$

This proves (ii).

We prove (iii). Again the issue is to compare surfaces in different metrics. In the case that $Y_{1}\left(t_{n}\right)$ is not a flat cylinder, let $\alpha$ any closed geodesic of $q_{1, \infty}$ in $Y_{\infty}$. By (15)

$$
\int_{F_{n}(\alpha)} d \nu_{j}\left(t_{n}\right) \rightarrow 0
$$


where $\nu_{j}\left(t_{n}\right)$ is the push forward measure of $\nu_{j}$ under $f_{t_{n}}$. But this is then also true for the geodesic in the class of $F_{n}(\alpha)$. Then (iii) holds by Lemma 2. If $Y_{1}\left(t_{n}\right)$ is a flat annulus we know that the $\nu_{j}\left(t_{n}\right)$ measure of any horizontal segment crossing $Y_{1}\left(t_{n}\right)$ is bounded by $\epsilon$. We now apply Lemma 3 to give the desired bound on the area.

Proof of Theorem A. We begin by assuming that the horizontal foliations of $q_{1}$ and $q_{2}$ coincide. Without loss of generality we can assume that for some minimal component $\Omega$ we have $a_{1}>0$ and $b_{1}=0$. It suffices to show that for any $M>0$ and for any sequence $t_{n} \rightarrow \infty$, for $t_{n}$ sufficiently large there is a simple closed curve $\gamma\left(t_{n}\right)$ with

$$
\frac{\operatorname{Ext}_{X_{2}\left(t_{n}\right)}\left(\gamma\left(t_{n}\right)\right)}{\operatorname{Ext}_{X_{1}\left(t_{n}\right)}\left(\gamma\left(t_{n}\right)\right)}>M
$$

For all $\epsilon>0$ small we now apply Proposition 1 . We find a fixed constant $B$ such that for $t_{n}$ sufficiently large, the subsurface $Y\left(t_{n}\right)$, given by that Proposition, satisfies

$$
\begin{gathered}
\operatorname{Area}_{q_{1}\left(t_{n}\right)}\left(Y\left(t_{n}\right)\right)>B \\
\text { Area }_{q_{2}\left(t_{n}\right)}\left(Y\left(t_{n}\right)\right)<\epsilon
\end{gathered}
$$

and

$$
\operatorname{Ext}_{X_{1}\left(t_{n}\right)}\left(\partial Y\left(t_{n}\right)\right) \leq \epsilon / M
$$

If $\left|\partial Y\left(t_{n}\right)\right|_{q_{2}\left(t_{n}\right)} \geq \sqrt{\epsilon}$, then $\operatorname{Ext}_{X_{2}\left(t_{n}\right)}\left(\partial Y\left(t_{n}\right)\right) \geq \epsilon$, and we are done; we may choose $\gamma\left(t_{n}\right)=\partial Y\left(t_{n}\right)$. Thus assume

$$
\left|\partial Y\left(t_{n}\right)\right|_{q_{2}\left(t_{n}\right)}<\sqrt{\epsilon} .
$$

If $Y\left(t_{n}\right)$ is not a flat cylinder, for $\epsilon$ small enough, we can apply Lemma 6 to find a bounded length $\left(q_{1}\left(t_{n}\right), \delta\right)$-almost vertical curve $\gamma_{n} \subset Y\left(t_{n}\right)$ and then Lemma 7 , which says that $\gamma_{n}$ has the desired property (19).

Thus assume $Y\left(t_{n}\right)$ is a flat cylinder. Let $\beta_{n}$ be a core curve of $Y\left(t_{n}\right)$ with $t_{n}$ chosen so that $\left|\beta_{n}\right|_{q_{1}\left(t_{n}\right)}<\epsilon$. Fix some $\delta_{0}>0$. Suppose first that $\beta_{n}$ is $\left(q_{1}\left(t_{n}\right), \delta_{0}\right)$-almost vertical. The reciprocal of the modulus of the cylinder is an upper bound for $\operatorname{Ext}_{X_{1}\left(t_{n}\right)}(\alpha)$, and we have

$$
\operatorname{Ext}_{X_{1}\left(t_{n}\right)}\left(\beta_{n}\right) \leq \frac{\left|\beta_{n}\right|_{q_{1}\left(t_{n}\right)}^{2}}{\operatorname{Area}_{q_{1}\left(t_{n}\right)}\left(Y\left(t_{n}\right)\right)} \leq \frac{\left|\beta_{n}\right|_{q_{1}\left(t_{n}\right)}^{2}}{B}
$$

We now want to estimate $\operatorname{Ext}_{X_{2}\left(t_{n}\right)}\left(\beta_{n}\right)$. The assumption that $\beta_{n}$ is $\left(q_{1}\left(t_{n}\right), \delta_{0}\right)$-almost vertical and since vertical lengths coincide, implies by (8) that

$$
\left|\beta_{n}\right|_{q_{2}\left(t_{n}\right)}>\frac{\delta_{0}\left|\beta_{n}\right|_{q_{1}\left(t_{n}\right)}}{\left(1+\delta_{0}\right)} .
$$

Now there is an annulus $A\left(t_{n}\right)$ which is a union of the flat annulus $Y\left(t_{n}\right)$ and an expanding annulus $Y^{\prime}\left(t_{n}\right)$. By Proposition 1 the $q_{2}\left(t_{n}\right)$-area of $Y\left(t_{n}\right)$ is bounded by $c \epsilon$ for some fixed 
$c>0$. By Lemma 1 and since extremal length and hyperbolic length are asymptotic, there are constants $c^{\prime}, c^{\prime \prime}>0$ depending on $c$ and $\delta_{0}$ but independent of $\epsilon$ and $t_{n}$ such that

$$
\begin{gathered}
\operatorname{Ext}_{X_{2}\left(t_{n}\right)}\left(\beta_{n}\right) \geq \frac{c^{\prime}}{\operatorname{Mod}\left(Y\left(t_{n}\right)\right)+\operatorname{Mod}\left(Y^{\prime}\left(t_{n}\right)\right)} \geq \\
\frac{c^{\prime}}{\frac{\operatorname{Area}_{q_{2}}\left(Y\left(t_{n}\right)\right)}{\left|\beta_{n}\right|_{q_{2}\left(t_{n}\right)}^{2}}-\log \left|\beta_{n}\right|_{q_{2}\left(t_{n}\right)}} \geq \frac{\epsilon}{\frac{\epsilon}{\left|\beta_{n}\right|_{q_{1}\left(t_{n}\right)}^{2}}-\log \left|\beta_{n}\right|_{q_{1}\left(t_{n}\right)}} .
\end{gathered}
$$

Comparing with (20) we see that for $\epsilon$ small enough, $\beta_{n}$ is a curve that satisfies (19).

Suppose now the core curve $\beta_{n}$ of $Y\left(t_{n}\right)$ is not $\left(q_{1}\left(t_{n}\right), \delta_{0}\right)$ almost vertical. If $Y\left(t_{n}\right)$ is nonseparating, choose a nontrivial isotopy class of arcs in the complement of $Y\left(t_{n}\right)$ joining the top and bottom of $Y\left(t_{n}\right)$. If $Y\left(t_{n}\right)$ is separating, choose two nontrivial isotopy classes, one that joins the top of $Y\left(t_{n}\right)$ to itself and the other which joins the bottom to itself. These families can be chosen to lie in the thick part of the surface $X_{1}\left(t_{n}\right)$ and as such have extremal length bounded independently of $t_{n}$. In the first case we also take a family of arcs $\alpha_{n}$ crossing $Y\left(t_{n}\right)$ that intersect a fixed perpendicular at most once. The arcs $\alpha_{n}$ are $\left(q_{1}\left(t_{n}\right), \frac{1}{2 \delta_{0}}\right)$ almost vertical. In the second case we take a pair of (families of) arcs crossing $Y\left(t_{n}\right)$ which are $\left(q_{1}\left(t_{n}\right), \frac{1}{2 \delta_{0}}\right)$ almost vertical. Let $\delta^{\prime}=\frac{1}{2 \delta_{0}}$. Now we can form a closed curve $\gamma_{n}$ as a concatenation of an arc outside $Y\left(t_{n}\right)$ and an arc $\alpha_{n}$, or, in the separating case, a pair of arcs outside and a pair of arcs crossing. For some constant $c^{\prime}$, again by Theorem 1

$$
\operatorname{Ext}_{X_{1}\left(t_{n}\right)}\left(\gamma_{n}\right) \leq c^{\prime} \operatorname{Ext}_{X_{1}\left(t_{n}\right)}\left(\alpha_{n}\right)=c^{\prime} \frac{\inf \left|\alpha_{n}\right|_{q_{1}\left(t_{n}\right)}^{2}}{\operatorname{Area}_{q_{1}\left(t_{n}\right)}\left(Y\left(t_{n}\right)\right)} \leq c^{\prime} \frac{\inf \left|\alpha_{n}\right|_{q_{1}\left(t_{n}\right)}^{2}}{B} .
$$

On the other hand, the curves $\gamma_{n}$ formed this way are longer than the arcs $\alpha_{n}$ crossing the cylinder. Since the $\operatorname{arcs} \alpha_{n}$ are $\left(q_{1}\left(t_{n}\right), \delta^{\prime}\right)$ almost vertical, we have

$$
\operatorname{Ext}_{X_{2}\left(t_{n}\right)}\left(\gamma_{n}\right) \geq \operatorname{Ext}_{X_{2}\left(t_{n}\right)}\left(\alpha_{n}\right)=\frac{\left|\alpha_{n}\right|_{q_{2}\left(t_{n}\right)}^{2}}{\operatorname{Area}_{q_{2}\left(t_{n}\right)}\left(Y\left(t_{n}\right)\right)} \geq \frac{\delta^{\prime}\left|\alpha_{n}\right|_{q_{1}\left(t_{n}\right)}^{2}}{c \epsilon\left(1+\delta^{\prime}\right)},
$$

and we are done for $\epsilon$ small enough. We have proven the theorem in the case that the horizontal foliations coincide.

Now consider the general case where the horizontal foliations of $q_{1}$ and $q_{2}$ are distinct. Pick a quadratic differential $q_{3}$ with the same vertical foliation as $q_{1}$ and the same horizontal foliation as $q_{2}$. The rays determined by $q_{3}$ and $q_{2}$ diverge by what was already proved. The rays determined by $q_{1}$ and $q_{3}$ stay bounded distance apart as a special case of Ivanov's result [5].

Proof of Theorem B. Denote the foliations simply by $F_{1}, F_{2}$. The first case is if the minimal components, if any, coincide. Since the foliations are not topologically equivalent, and yet have 0 intersection number, there must be some curve $\beta$ which is a core curve of a flat cylinder with respect to one quadratic differential, say $q_{1}$, but is not the core curve of a flat cylinder of $q_{2}$. Since $\beta$ is isotopic to the core curve of a flat annulus of $q_{1}$ we have

$$
\operatorname{Ext}_{X_{1}(t)}(\beta) \leq c e^{-2 t}
$$


for some $c$. Since $\beta$ is not a subset of a minimal component of $F_{2}$, we must have $\beta \subset \Gamma_{q_{2}}$, the critical graph of $q_{2}$. Now the length of $\beta$ in the metric of $q_{2}(t)$ satisfies $|\beta|_{q_{2}(t)}=$ $|\beta|_{q_{2}} e^{-t} \rightarrow 0$. Now $\beta$ determines an expanding annulus. We apply the upper bound for the modulus of that annulus as given in Lemma 1 and hence the lower bound for extremal length to say that

$$
\frac{\operatorname{Ext}_{X_{2}(t)}(\beta)}{\operatorname{Ext}_{X_{1}(t)}(\beta)} \rightarrow \infty .
$$

The second case is if one of the foliations, say $F_{1}$, has a minimal component $\Omega_{1}$ which is not a minimal component of $F_{2}$. Since $i\left(F_{1}, F_{2}\right)=0$, every curve $\beta \subset \Omega_{1}$ satisfies $i\left(F_{2}, \beta\right)=0$, so that $\beta \subset \Gamma_{q_{2}}$, the critical graph of $q_{2}$. Since $\beta \subset \Omega_{1}$, we have $h_{q_{1}}(\beta)>0$ and so the flat length of $\beta$ with respect to $q_{t}$ satisfies

$$
|\beta|_{q_{1}(t)} \geq h_{q_{1}}(\beta) e^{t}
$$

This gives

$$
\operatorname{Ext}_{X_{1}(t)}(\beta) \geq h_{q_{1}}^{2}(\beta) e^{2 t} .
$$

It suffices to find an upper bound for $\operatorname{Ext}_{X_{2}(t)}(\beta)$. Now $\beta$ is either on the boundary of a minimal component $\Omega_{2}$ of $F_{2}$ or is on the boundary of a flat cylinder. In either case it determines a maximal expanding annulus $A$. Since $\Omega_{2}$ is minimal, the shortest saddle connection $\gamma(t)$ contained in $\Omega_{2}$ satisfies $|\gamma(t)|_{q_{2}} \rightarrow \infty$ as $t \rightarrow \infty$ and hence

$$
e^{t}|\gamma|_{q_{2}(t)} \rightarrow \infty
$$

This means that for a constant $c>0, d(A) \geq c e^{-t}$. Since $|\beta|_{q_{2}(t)} \asymp e^{-t}$, by Lemma 1 the modulus $A$ is bounded below and so the extremal length of $\beta$ is bounded above.

Proof of Theorem $C$. We note that each $\gamma_{n}^{j}$ may itself be a multicurve. Fix a finite set of curves $\alpha_{1} \ldots, \alpha_{N}$ such that the intersection of any measured foliaiton with these curves determines the foliation. Choose a unit area quadratic differential $q$ on some surface $X$ whose vertical foliation is $\left[F, \sum_{i=1}^{p} \nu_{i}\right]$. Denote by $|d y|$ the measure on the corresponding horizontal foliation. Let $X(t)$ be the corresponding ray. For any sequence of times $t_{n} \rightarrow \infty$ by Proposition 1 there is $B>0$ and a collection of disjoint domains $Y_{1}\left(t_{n}\right), \ldots, Y_{p}\left(t_{n}\right)$ such that the area of $Y_{i}\left(t_{n}\right)$ with respect to the measure $d \nu_{i}|d y|$ is at least $B$. Suppose first that $Y_{i}\left(t_{n}\right)$ is not a cylinder. By the first part of Lemma 6 we may pick a $\left(q\left(t_{n}\right), \delta\right)$ almost vertical curve $\gamma_{i}\left(t_{n}\right) \subset Y_{i}\left(t_{n}\right)$ of length at most $D$. We claim that $\gamma_{i}\left(t_{n}\right) \rightarrow\left[F, \nu_{i}\right]$. As before, let $\Lambda_{i}\left(t_{n}\right)$ be the image of the generic points inside $Y_{i}\left(t_{n}\right)$; generic with respect to the transversals for the set of $\alpha_{i}$. The generic points are dense, and $\gamma_{i}\left(t_{n}\right)$ is a union of a bounded number of saddle connections, so we can find a bounded collection $\left\{\omega_{j}\left(t_{n}\right)\right\}_{j=1}^{m}$ of vertical segments beginning at generic points satisfying the second conclusion of Lemma 6 . By construction of the $\omega_{j}\left(t_{n}\right)$, for any fixed $\alpha_{k}$,

$$
\frac{i\left(\gamma_{i}\left(t_{n}\right), \alpha_{k}\right)}{\sum_{j=1}^{m} i\left(\omega_{j}\left(t_{n}\right), \alpha_{k}\right)} \rightarrow 1
$$


Since $\omega_{j}\left(t_{n}\right)$ is a vertical segment through a generic point,

$$
\frac{\operatorname{card}\left(\omega_{j}\left(t_{n}\right) \cap \alpha_{k}\right)}{\left|\omega_{j}\left(t_{n}\right)\right|_{q(0)}} \rightarrow \nu_{i}\left(\alpha_{k}\right)
$$

Summing over all $1 \leq j \leq m$ we have

$$
\frac{i\left(\gamma_{i}\left(t_{n}\right), \alpha_{k}\right)}{\sum_{j=1}^{m}\left|\omega_{j}(n)\right|_{q(0)}} \rightarrow \nu_{i}\left(\alpha_{k}\right) .
$$

However

$$
\frac{\sum_{j=1}^{m}\left|\omega_{j}(n)\right|_{q(0)}}{v_{q(0)}\left(\gamma_{i}\left(t_{n}\right)\right)} \rightarrow 1,
$$

and so if we let $s_{n}=\frac{1}{v_{q(0)}\left(\gamma_{i}\left(t_{n}\right)\right)}$ then we have for each $k$,

$$
\lim _{n \rightarrow \infty} s_{n} i\left(\gamma_{i}\left(t_{n}\right), \alpha_{k}\right) \rightarrow \nu_{i}\left(\alpha_{k}\right)
$$

and we are done.

Finally suppose $Y_{i}\left(t_{n}\right)$ is a flat cylinder with core curve $\gamma_{i}\left(t_{n}\right)$. As in the proof of Proposition 1 we can assume that $t_{n}$ is chosen so that $\gamma_{i}\left(t_{n}\right)$ is $\left(q\left(t_{n}\right), 2\right)$-almost vertical. As in that argument we find a dense set of generic points $\Lambda_{i}^{T_{0}}$, generic for the transversals to the $\alpha_{i}$. We then can find vertical segments $\omega_{j}\left(t_{n}\right)$ through generic points such that (21) holds and the rest of the proof is the same.

\section{REFERENCES}

[1] L Ahlfors Lectures on Quasiconformal Mappings, University Lecture Series, AMS, 2nd edition, 2006

[2] L Bers Spaces of degenerating Riemann surfaces. Discontinuous groups and Riemann surfaces, Annals of Mathematics Studies 79, Princeton University Press, Princeton, New Jersey, 1974

[3] Y.Choi, K.Rafi, C.Series Lines of minima and Teichmüller geodesics To appear, Geometric and Functional Analysis

[4] A.Fathi, F.Laudenbach, V.Poenaru Travaux de Thurston sur les surfaces, Asterisque, Vol. 66-67 (1979)

[5] N.Ivanov Isometries of Teichmüller spaces from the point of view of Mostow rigidity, Topology, ergodic theory, real algebraic geometry, ed. by V. Turaev and A. Vershik, Amer. Math. Soc. Transl. Ser. 2, V. 202, American Mathematical Society, 2001, 131-149.

[6] S. Kerckhoff The asymptotic geometry of Teichmüller space, Topology, Vol. 19, 23-41.

[7] H. Masur On a class of geodesics in Teichmüller space Annals of Math., Vol. 102, No. 2 (1975), 205-221.

[8] H.Masur Uniquely ergodic quadratic differentials, Comment. Math. Helvetici Vol. 55 (1980) 255-266

[9] H.Masur Hausdorff dimension of the set of nonergodic foliations of a quadratic differential Duke Math. J. Vol 66 (1992) 387-442

[10] H.Masur, S.Tabachnikov, Rational Billiards and Flat Structures, Handbook of Dynamical Systems, B. Hasselblatt, A. Katok eds. Elsevier (2002), 1015-1089

[11] H.Masur, Interval exchange transformations and measured foliations Ann. of Math. Vol 115 (1982) $169-200$

[12] B Maskit, Comparison of hyperbolic and extremal lengths, Ann. Acad. Sci. Fenn. Ser. A I Math. 10 (1985), 381-386. MR 87c:30062

[13] Y Minsky, Extremal length estimates and product regions in Teichmüller space, Duke Math. J. Volume 83, Number 2 (1996), 249-286 
[14] Y Minsky Harmonic maps, length, and energy in Teichmüller space, J. Differential Geometry, Volume 35 (1992), 151-217

[15] K Rafi Thick-Thin decomposition for quadratic differentials, Math. Res. Lett. 14 (2007), no. 2, 333-341

Anna Lenzhen:

Dept. of Mathematics

University of Michigan

Ann Arbor, Michigan, 48109

E-mail: alenzhen@umich.edu

Howard Masur:

Dept. of Mathematics, University of Chicago

Chicago, IL 60637

E-mail: masur@math.uchicago.edu 